\title{
DSM EXTRACTION AND EVALUATION FROM GEOEYE-1 STEREO IMAGERY
}

\author{
M.M. Saldaña, M.A.* Aguilar, F.J. Aguilar, I. Fernández
}

High School Engineering, Department of Agricultural Engineering, Almería University, 04120 La Cañada de San Urbano, Almería, Spain - marsalda86@hotmail.com, maguilar@ual.es, faguilar@ual.es, ismaelf@ual.es

Commission IV, WG IV/3

KEY WORDS: DEM/DTM, Accuracy, Imagery, Pushbroom, High resolution, Satellite

\begin{abstract}
:
The newest very high resolution (VHR) commercial satellites, such as GeoEye-1 or WorldView-2, open new possibilities for cartographic applications, orthoimages generation and extraction of Digital Surface Models (DSMs). These DSMs are generated by image matching strategies from VHR satellite stereopairs imagery, reconstructing the 3D surface corresponding to the first surface view of the earth containing both microrelief (buildings, trees and so on) and bare terrain. The main aim of this work is to carry out an accuracy assessment test on the DSMs extracted from a GeoEye-1 stereopair captured in August 2011. A LiDAR derived DSM taken at the same month that the satellite imagery was used as ground truth. The influence of factors such as number of Ground Control Points (GCPs), sensor models tested and the geoid employed to transform the ellipsoid to orthometric heights were going to be evaluated. In this way, different sets of GCPs ranging from 7 to 45, two sensor models and two geoids (EGM96 and EGM08, the last adapted for Spain vertical network by the Spanish's National Geographic Institute) were tested in this work. The photogrammetric software package used was OrthoEngine from PCI Geomatica v. 10.3.2. OrthoEngine implements both sensor models tested: (i) the physical model developed by Toutin (CCRS) and, (ii) the rational function model using rational polynomial coefficients supplied by the vendor and later refined by means of the zero order linear functions (RPC0). When high accurate and well-distributed GCPs were used, the planimetric and vertical accuracies of DSMs generated from the GeoEye-1 Geo stereopair were always better than $0.5 \mathrm{~m}$. Using only 7 GCPs and RPC0, a vertical accuracy around $0.43 \mathrm{~m}$ measured as standard deviation was attained. The geoid used by OrthoEngine (EGM96) produced similar results that the EGM08 adapted for Spain vertical network.
\end{abstract}

\section{INTRODUCTION}

Digital Elevation Models (DEMs) and Digital Surface Models (DSMs) have large relevance in some territorial applications such as modeling water flow, mass movement, topographic mapping, Geographic Information Systems, natural hazards, spatial and temporal change detection, feature extraction, visualization and many others (Deilami and Hashim, 2011). For decades the terrestrial surveying techniques and aerial images were the only approaches available for generating DEM. However, with the launch of the first very high resolution (VHR) commercial satellites, IKONOS in September 1999 and QuickBird in October 2001, conventional aerial photogrammetric mapping at large scales began to have serious competitors. Besides, many new VHR satellites, capable of capturing panchromatic imagery of the land surface with Ground Sample Distance (GSD) of $1 \mathrm{~m}$ and even lower, such as EROS B1, Resurs DK-1, KOMPSAT-2, IRS Cartosat 2, WorldView-1, have been launched during 2006 and 2007, and they are offering to their customers very high resolution imagery of the Earth, with a very shortly revisit time. The rapid increase of commercial VHR satellites in the last years has resulted in improvements in resolution, availability and cutting down on cost.

A new commercial VHR satellite called GeoEye-1 (GeoEye, Inc., 2009) was launched in 2008. Nowadays, it is the commercial satellite with the highest geometric resolution, in panchromatic (0.41 $\mathrm{m}$ at nadir) and in multispectral $(1.65 \mathrm{~m}$ at nadir) products. However, image products from GeoEye-1 have to be down-sampled to $0.5 \mathrm{~m}$ and $2 \mathrm{~m} \mathrm{GSD}$, panchromatic (PAN) and multispectral (MS) respectively, for commercial sales, as a requirement levied by the U.S.

\footnotetext{
${ }^{*}$ Corresponding author
}

The new VHR satellite images from GeoEye-1 open new possibilities for cartographic applications and extraction of DSMs. These DSMs are generated by image matching strategies from VHR satellite stereopairs imagery. In this way, the first geopositioning accuracy results attained from GeoEye-1 PAN stereopairs were superior enough to those obtained from older satellites such as IKONOS or QuickBird. Fraser and Ravanbakhsh (2009) achieved vertical and horizontal accuracies of $0.25 \mathrm{~m}$ and $0.10 \mathrm{~m}$ respectively using a stereopair of GeoEye-1, whereas Mitchell and MacNabb (2010) reported a vertical Root Mean Square Error (RMSEz) of $0.25 \mathrm{~m}$ by using a LiDAR derived DEM as ground truth.

The main aim of this work is to carry out an accuracy assessment test on the DSMs extracted from a GeoEye-1 stereopair. A highly accurate LiDAR derived DSM has been used as ground truth. In this assay, the impact of three factors on DSM accuracy is going to be evaluated:

(i) The sensor model used in the triangulation or bundle adjustment. Concretely, two sensor models are going to be tested. The first of them is a $3 \mathrm{D}$ rational functions with vendor supplied rational polynomial coefficients (RPCs) and refined with Ground Control Points (GCPs). The second is a rigorous or physic model.

(ii) The number of GCPs used for computing both sensor models. Different sets of 7,12 and 45 GCPs are going to be tested.

(iii) The influence of the geoid used for change the ellipsoidic heights to orthometric heights was also tested. 


\section{STUDY SITE AND DATA SETS}

\subsection{Study site}

The study area comprises a heavily developed coastal fringe located at Almería (Mediterranean Sea, Southern Spain), approximately $11 \mathrm{~km}$ long and $775 \mathrm{~m}$ wide (Fig. 1 and 2). The working area is situated between the harbours of Garrucha and Villaricos, and it is is centred on the UTM 30N WGS84 coordinates (Easting and Northing) of $605870 \mathrm{~m}$ and 4119869 $\mathrm{m}$. The study area presents a mean elevation close to $7 \mathrm{~m}$, ranging from $0 \mathrm{~m}$ to $55 \mathrm{~m}$ above mean sea level.

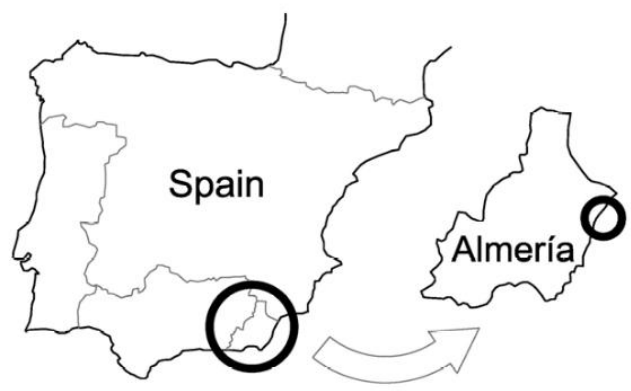

Figure 1. Location of the working area.

\subsection{GeoEye-1 Stereopair}

A panchromatic stereopair of GeoEye-1 (Stereo Geo PAN) comprising two images taken on the same orbital pass was acquired. It was captured in reverse scan mode on August 27, 2011 with $0.5 \mathrm{~m}$ GSD.

The forward looking image had a collection azimuth of $183.62^{\circ}$ and an elevation of $66.85^{\circ}$, while the corresponding values for the backward looking image were $40.39^{\circ}$ and $81.47^{\circ}$. This produced a base/height ratio of 0.57 , which is suitable for cartographical mapping and DSM extraction. Besides, according with Li et al. (2007), the convergence angle between these two images was 30.4 degrees. The characteristics of the GeoEye-1 images are shown in Table 1.

\begin{tabular}{|l|c|c|}
\hline Image & Forward & Backward \\
Product & GEO_Stereo & GEO_Stereo \\
Acquisition Date & $27 / 08 / 11$ & $27 / 08 / 11$ \\
Cloud Cover (\%) & 0 & 0 \\
Collection Azimuth & $183.62^{\circ}$ & $40.39^{\circ}$ \\
Collection Elevation & $66.85^{\circ}$ & $81.47^{\circ}$ \\
Sun Angle Azimuth & $144.40^{\circ}$ & $144.06^{\circ}$ \\
Sun Angle Elevation & $58.36^{\circ}$ & $58.27^{\circ}$ \\
Pixel size & $0.5 \mathrm{~m}$ & $0.5 \mathrm{~m}$ \\
Nominal GSD Along Scan & $0.440 \mathrm{~m}$ & $0.413 \mathrm{~m}$ \\
Nominal GSD Cross Scan & $0.479 \mathrm{~m}$ & $0.415 \mathrm{~m}$ \\
Product Pixel Size & $0.5 \mathrm{~m}$ & $0.5 \mathrm{~m}$ \\
\hline
\end{tabular}

Table 1. Characteristics of the GeEye-1 Stereopair.

\subsection{Ground Points}

The ground points coordinates were obtained by differential global positioning system (DGPS) using a total GPS Topcon HiPer PRO station working in real time kinematic mode (RTK).
The coordinates of 120 ground points, located on well-defined features and homogeneously distributed over the study area, were measured with reference to the European Terrestrial Reference System 1989 (ETRS89) and UTM projection. The vertical datum took the geoid as the reference surface, adopting the mean sea level in the calm seas of Alicante (Spain) as the null orthometric height point.

From all ground points, 51 were selected as GCPs and the remained 69 were used as Independent Check Points (ICPs). Three different sets of 7 GCPs, 12GCPs and 45 GCPs were chosen from the 51 initial GCPs (Fig. 2).

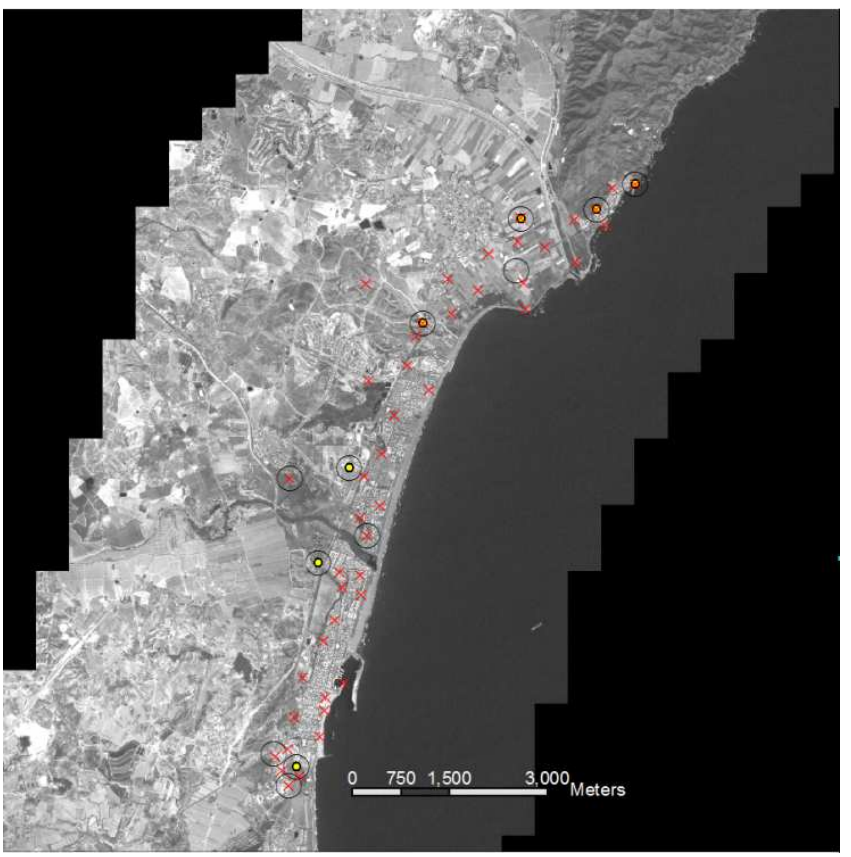

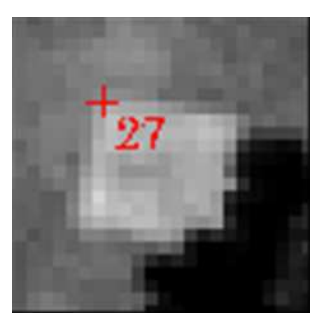

b) a)

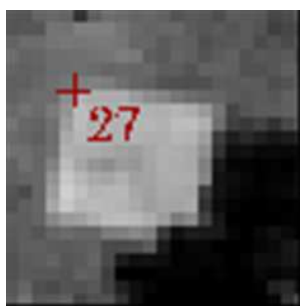

c)
Figure 2. a) Distribution of 45 GCPs (red crosses), 12 GCPs (black circles) and $7 \mathrm{GCPs}$ (yellow point) overlaid on the GeoEye-1 panchromatic image. b) GCP number 27 (forward GEO stereo). c) GCP number 27 (backward GEO stereo).

For the combinations of 7 and 12 GCPs, the points were all located on the ground. In the case of 45 GCPs, some GCPs were located on roofs, buildings, or other structures with elevation. It is noteworthy that at the points defined on structures with elevation, a little error in the introduction of photo coordinates could provoke an important error in Z. All cases, the same GCPs were pointed in forward and backward GEO stereopair.

A high number of ICPs were used in this work because the reliability of the accuracy estimations depends, among other variables, on the number of ICPs used to compute them (e.g., Aguilar et al., 2008). The National Standard for Spatial Data Accuracy (NSSDA) by the Federal Geographic Data Committee (FGDC, 1998) and the Joint Research Centre, European 
Commission (JRC, 2008) recommend the use of a minimum of 20 ICPs, which should be, at least, three times more accurate than the final product specification.

\subsection{Sensor model used}

Several sensor models can be used to correct satellite imagery. The photogrammetric software package used in this work was OrthoEngine from PCI Geomatica v. 10.3.2, developed by PCI Geomatics (PCI Geomatics, Richmond Hill, Ontario, Canada). OrthoEngine implements the two models tested in this work.

(i) A rigorous, physical or parametric model based on a standard photogrammetric approach model developed by Toutin at the Canada Centre for Remote Sensing (Toutin, 2003) called CCRS here. This physical model was developed for medium-resolution sensors in the visible and infra-red as well as in the microwave (Toutin, 1995) and it was later adapted for satellite data of very high resolution (Toutin, 2004). CCRS model was calculated using both 12 and 45 GCPs.

(ii) A third order 3D rational functions with vendor's rational polynomial coefficients (RPCs) data and refined by a zero order polynomial adjustment (RPC0). OrthoEngine's RPC indirect method is based on the block adjustment method published by Grodecki and Dial (2003) for image space (equation 1), where $a_{0}$ to $a_{5}$ and $b_{0}$ to $b_{5}$ are the adjustment parameters of an image, $\Delta x$ and $\Delta y$ express the discrepancies between the line measured and the sample coordinates for the new GCPs in the image space $\left(x^{\prime}, y^{\prime}\right)$ and the RPCs projected coordinates for the same $\operatorname{GCPs}(x, y)$.

$$
\begin{aligned}
& \Delta x=x^{\prime}-x=a_{0}+a_{1} x+a_{2} y+a_{3} x y+a_{4} x^{2}+a_{5} y^{2} \\
& \Delta y=y^{\prime}-y=b_{0}+b_{1} x+b_{2} y+b_{3} x y+b_{4} x^{2}+b_{5} y^{2}
\end{aligned}
$$

For GeoEye-1 images, a zero order polynomial adjustment is required to achieve the best results (e.g., Fraser and Ravanbakhsh, 2009; Aguilar et al., 2012). In this work, RPC0 model was computed using both 7 and 12 GCPs.

\subsection{Geoid}

The DSM extracted from an OrthoEngine project using any rational function model is above ellipsoid. As ground points and the LiDAR derived DSM used as ground truth had orthometric heights, a geoid was needed. The influence of the geoid used for changing the ellipsoidic heights to orthometric heights was studied. In this work, the global Earth Gravitational Model of 1996 geoid (EGM96) and the new Earth Gravitational Model of 2008 (EGM2008), adapted for Spain vertical network by the Spanish's National Geographic Institute were compared.

\subsection{LiDAR derived DSM}

A high accuracy and resolution LiDAR derived DSM (LiDAR 2011) was used in this work as ground truth. It was taken on August $30^{\text {th }}, 2011$, as a combined photogrammetric and LiDAR survey at a flying height above ground of approximately $350 \mathrm{~m}$. A helicopter laser scanner developed by AeroLaser System S.L. based on a Riegl LMS Q240i was used. 30 degrees FOV was used, obtaining an average point density better than 2 points $/ \mathrm{m}^{2}$.

This DSM was compared, only in areas without buildings, trees, greenhouses or other structures with elevation, with a LiDAR derived DEM with a grid spacing of $1 \mathrm{~m}$ and taken on August 28th, 2009, with a Leica ALS60 airborne laser scanner (35 degrees field of view). The estimated vertical accuracy of the
LiDAR derived DEM from 2009 (LiDAR 2009) computed from 62 DGPS ICPs took a value of $8.9 \mathrm{~cm}$.

The comparison between DSM LiDAR 2011 and DEM LiDAR 2009 presented an average distance of $3.7 \mathrm{~cm}$ and a standard deviation of $9.5 \mathrm{~cm}$. These results were good enough being this reason why the DSM LiDAR 2011 was used as ground truth in this work.

\subsection{Control areas}

The accuracy assessment tests on the DSMs extracted from GeoEye-1 stereopair was carried out using the DSM LiDAR 2011 as ground truth. For obtaining reliable results, it is necessary to compare both DSMs (LiDAR and GeoEye-1) only in well-defined areas and without alterations of heights. Because of this reasons, the comparison between DSMs was computed on 34 sports areas such as football pitch, basketball or tennis courts and similar, with a total area close to $30000 \mathrm{~m}^{2}$. These areas were well-distributed over the working area. Within these areas, 78508 points were measured by AeroLaser's LiDAR, and around to 30000 points were extracted from the GeoEye-1 stereopair derived DSMs.

\section{METHODOLOGY}

In order to obtain the aims of this work, the next steps were carried out.

\subsection{DSM extraction}

OrthoEngine from PCI Geomatica v. 10.3.2 was the software used for both sensor orientation and DSM extraction. The automated image matching or image correlation technique was used to extract matching points on the two overlapping images from a search for pixels of corresponding contrast and brightness. This procedure is based on a mean normalized crosscorrelation matching method with a multi-scale strategy to match the image using the statistics collected in the defined windows.

Only a few parameters can be adjusted by the user into OrthoEngine DSM extraction module, such as the sampling interval and the level of detail. For every DSM, the epipolar images were generated with $0.5 \mathrm{~m}$ GSD (down sample factor of 1). A high detail DSM with 32 bits and a pixel sampling factor of 2 were selected into OrthoEngine. Thus, DSMs with a resolution of $1 \mathrm{~m}$ were attained. Regarding the sensor model, number of GCPs and geoid used, the following six different strategies were carried out for extracting DSMs from the GeoEye-1 stereopair:

(i) Using RPC0 sensor model and 7 GCPs with orthometric heights (mean sea level, MSL). In this case, OrthoEngine had to transform these GCPs heights to ellipsoidic elevations using the EGM96 geoid. Then, DSM was extracted above ellipsoid, and after that, this DSM had to be converted to MSL using again EGM96.

(ii) Using RPC0 sensor model and 7 GCPs with ellipsoidic heights computed from the original GCPs with orthometric heights using the EGM2008 adapted for Spain. In this case, OrthoEngine didn't have to transform these GCPs heights for extracting the DSM above ellipsoid. Finally, this DSM was converted to MSL using the adapted EGM2008 by mean of a MATLAB code. 
(iii) Using RPC0 sensor model and 12 GCPs with orthometric heights.

(iv) Using RPC0 sensor model and 12 GCPs with ellipsoidic heights.

(v) Using CCRS sensor model and 12 GCPs with orthometric heights. It is noteworthy that with CCRS model is not possible working directly with ellipsoidic heights.

(vi) Using CCRS sensor model and 45 GCPs with orthometric heights.

\subsection{Accuracy assessment at ICPs}

The aim of this first accuracy assessment test was to observe the root mean square error (RMSE) along $X$ and $Y$ axes after the sensor orientation phase, depending on the sensor model, the number of GCPs and the type of GCPs heights used. RMSE $E_{x}$ and $\mathrm{RMSE}_{\mathrm{y}}$ were always computed for the same 69 ICPs for every project.

\subsection{LiDAR vs GeoEye-1 DSMs}

When the 34 sports areas were extracted from both LiDAR derived and GeoEye-1 DSMs, the comparison was carried out. For this task, RapidForm 2004 was the software used. The initial triangulation into RapidForm was carried out by deleting the faces with a maximum edge length larger than 5 meters.

When the triangulation was carried out, the differences between LiDAR and GeoEye-1 DSMs were extracted, proceeding to compute their distribution, mean and standard deviation computed.

\section{RESULTS AND DISCUSSION}

\subsection{Accuracy assessment at ICPs}

Table 2 shows the RMSE, $\mathrm{RMSE}_{\mathrm{y}}$ and $\mathrm{RMSE}_{2 \mathrm{~d}}$ values for the residuals computed at 69 ICPs along $X$ and $Y$ axes after the bundle adjustment of the GeoEye-1 stereopair.

\begin{tabular}{cclccc}
\hline $\begin{array}{c}\text { Sensor } \\
\text { Model }\end{array}$ & $\begin{array}{c}\text { N. } \\
\text { GCPs }\end{array}$ & Heights & RMSE $_{\boldsymbol{x}}$ & RMSE $_{\mathbf{y}}$ & RSME $_{\mathbf{2 d}}$ \\
\hline RPC0 & 7 & Orthometric & 0.284 & 0.269 & 0.391 \\
RPC0 & 7 & Ellipsoidic & 0.284 & 0.269 & 0.391 \\
RPC0 & 12 & Orthometric & 0.272 & 0.264 & 0.379 \\
RPC0 & 12 & Ellipsoidic & 0.272 & 0.265 & 0.379 \\
CCRS & 12 & Orthometric & 0.316 & 0.269 & 0.415 \\
CCRS & 45 & Orthometric & 0.274 & 0.247 & 0.369 \\
\hline
\end{tabular}

Table 2. Accuracy at the 69 ICPs for the sensor orientation phase. Values expressed in meters.

According to these results, the tested sensor models worked slightly better when more GCPs were used. On the other hand, the type of the GCPs heights (i.e., orthometric or ellipsoidic) didn't influence on the accuracy report. Bearing in mind that the report offered by OrthoEngine at the sensor model phase doesn't show the differences in $Z$, these results have to be taken warily and a second accuracy assessment is needed.

\subsection{DSM accuracy assessment}

Table 3 shows the statistics of residuals computed for every six GeoEye-1 derived DSMs when they were compared with the LiDAR ground truth.

Overall, RPC0 sensor model attained slightly better vertical accuracies for all DSMs generated from the GeoEye-1 stereopair. The rigorous sensor model (CCRS), as had already reported others authors, didn't work very well with GeoEye-1 images (Aguilar et al., 2012). Besides, CCRS need more GCPs than RPC0. However, in several studies, CCRS had proved to be more accurate and stable than RPC models for Cartosat-1 images (Kay and Zielinski, 2007) or QuickBird stereo images (Aguilar et al., 2007). On the other hand and regarding RPC0, neither the number of GCPs nor the use of more accurate geoid had any clear influence on the final results.

\begin{tabular}{cclcc}
\hline $\begin{array}{c}\text { Sensor } \\
\text { Model }\end{array}$ & N. GCPs & Heights & Mean (m) & Std (m) \\
\hline RPC0 & 7 & Orthometric & -0.118 & 0.444 \\
RPC0 & 7 & Ellipsoidic & -0.115 & 0.427 \\
RPC0 & 12 & Orthometric & -0.139 & 0.439 \\
RPC0 & 12 & Ellipsoidic & -0.123 & 0.456 \\
CCRS & 12 & Orthometric & 0.0246 & 0.465 \\
CCRS & 45 & Orthometric & -0.213 & 0.485 \\
\hline
\end{tabular}

Table 3. Statistic results on residuals elevations (LiDARGeoEye-1) for the DSMs with different combination of GCPs and different heights.

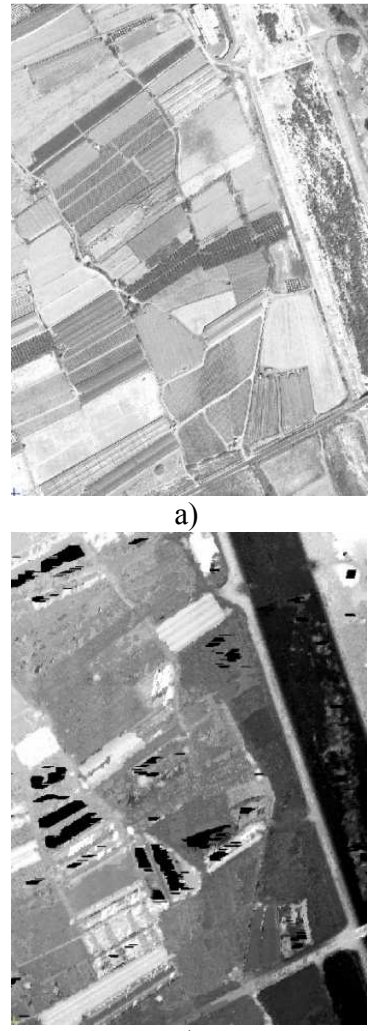

c)
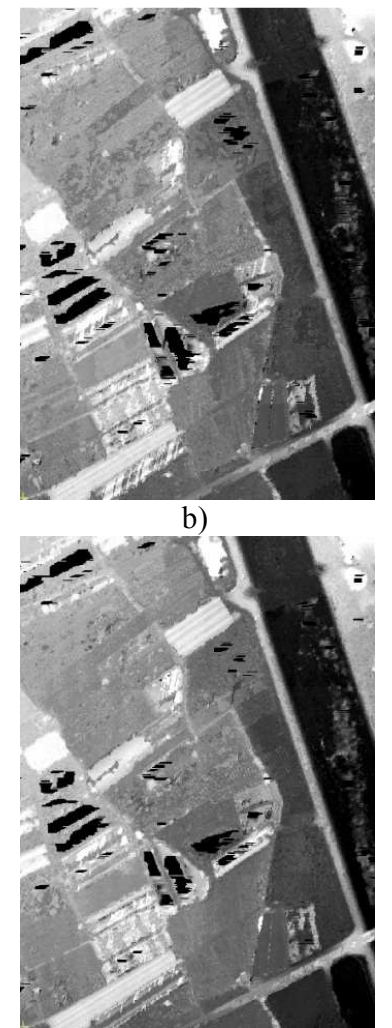

d)
Figure 3. DSMs from GeoEye-1 stereopair using orthometric heights in a zone near of the Almanzora river. a) Orthoimage from GeoEye-1, b) DSM with CCRS and 45 GCPs, c) DSM with RPC0 and 7 GCPs, d) DSM with RPC0 and 12 GCPs. 
All the strategies for obtaining DSMs from GeoEye-1 stereopair proved to be very sensitive to the variation of the quality of the GCPs. In fact, probably CCRS with 45 GCPs shows the worst results due to some of the GCPs were not posed directly on the bare earth. In these cases, a little pointing error could produce a high vertical error in the bundle adjustment. In spite of that, any visual differences between DSMs generated using orthometric heights could be observed (Fig. 3). In this figure, the failure values were located principally in greenhouse areas, where the image matching techniques did not work well.

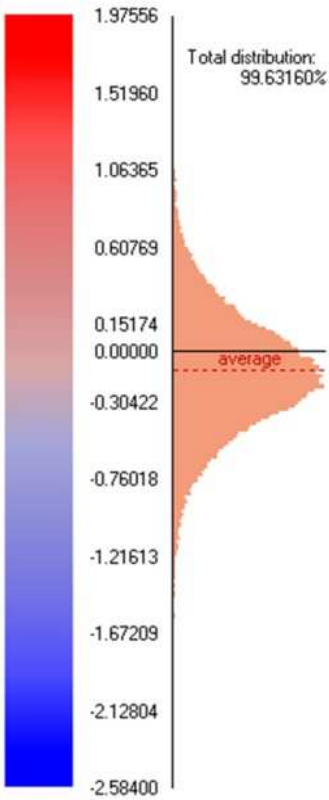

a)

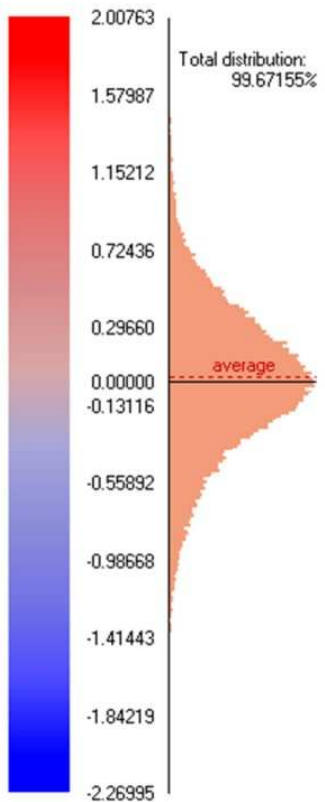

c)

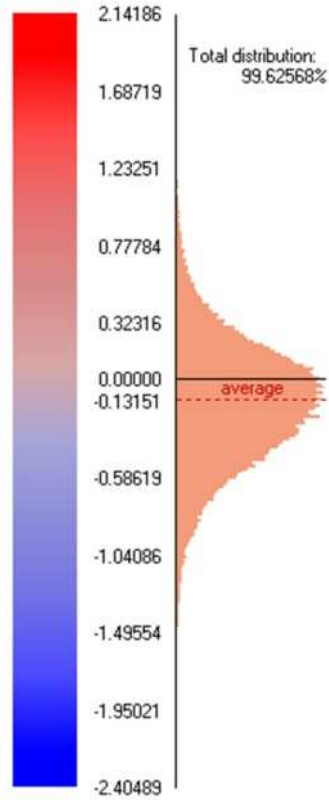

b)

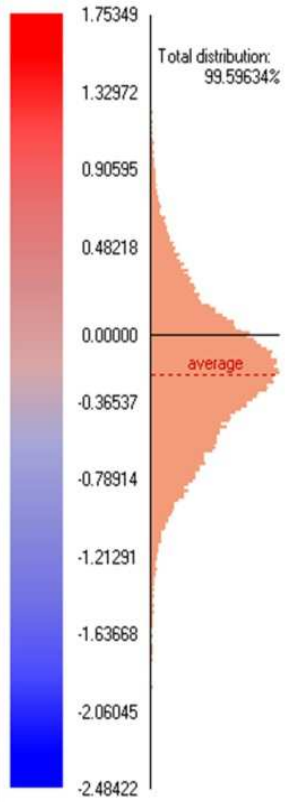

d)
Figure 4. Histograms showing differences in orthometric heights for the comparison between LiDAR DSM and GeoEye1 DSM. a) RPC0 and 7 GCPs, b) RPC0 and 12 GCPs, c) CCRS and 12 GCP), d) CCRS and 45 GCPS.
The total distributions corresponding to orthometric height differences presented in Figure 4 for the comparison between LiDAR 2011 and GeoEye-1's DSM, did not show any important difference. Only the average values were noteworthy. The existence of these possible systematic errors will be studied in further works.

The vertical accuracy results achieved by other authors working with GeoEye-1 stereopair were very changeable. Meguro and Fraser (2010) attained planimetric accuracy close to $0.38 \mathrm{~m}$ and $0.7 \mathrm{~m}$ as vertical accuracy at sensor orientation phase, whereas Fraser and Ravanbakhsh (2009) achieved vertical and horizontal accuracies of $0.25 \mathrm{~m}$ and $0.10 \mathrm{~m}$ respectively.

From a research of geolocation accuracy of GeoEye-1 stereo pair, Wang and Zhao (2011), using 5 GCPs and RPC0, reported $0.38 \mathrm{~m}$ as planimetric accuracy and $0.44 \mathrm{~m}$ as vertical accuracy, measured at 89 ICPs. They had also tested other four sensor models based on rational functions, although their results showed that the accuracy attained using any of the five compensation models was similar. In fact, when high accurate and well-distributed GCPs were used, the planimetry and height accuracy of GeoEye-1 stereo imagery pairs were always better than $0.5 \mathrm{~m}$.

\section{CONCLUSIONS}

A digital model of the elevation of the visible surface, known as Digital Surface Model (DSM), can be generated from a GeoEye-1 stereopair through the use of automatic image matching. For several applications, and due to the accuracy of these DSMs, they could be a direct competitors for the conventional aerial photogrammetric flights, and the choice about which product to use would be purely economic. Overall, when the VHR satellites derived DSMs accuracies meet the customers' requirements, the cost is usually lower than the traditional aerial photogrammetry and the process for its generation is faster.

Two sensor models, one based on rational functions, being the other a physical approach, were tested. However, when high accurate and well-distributed GCPs were used, the attained accuracy was similar for every case. The planimetric and vertical accuracies of DSMs generated from the GeoEye-1 Geo stereopair were always better than its GSD (i.e., $0.5 \mathrm{~m}$ ).

The geoid used by OrthoEngine (EGM96) yielded similar results that the EGM2008 (National Geospatial Intelligence Agency, http://www.nga.mil) adapted for Spain vertical network (REDNAP) by the Spanish National Geographic Institute. Probably, the fact that our working area was very small did not help to accentuate the absolute differences between both geoids.

The best results, based on the accuracy assessment of the DSMs carried out in this work, were generated by RPC0 model using 7 GCPs well-defined and posed on the soil surface. In general, all 3D models from GeoEye-1 imagery were very accurate, but the pointing error of the GCPs had a great influence in the final results. 


\section{ACKNOWLODGEMENTS}

This work was supported by the Spanish Ministry for Science and Innovation (Spanish Government) and the European Union (FEDER founds) under Grant Reference CTM2010-16573 (http://www.ual.es/Proyectos/GEOEYE1WV2/index.htm). The authors also appreciate the support from Andalusia Regional Government, Spain, through the Excellence Research Project RNM-3575. Thanks are due to AeroLaser Systems S.L., an innovative company for their cooperation and expertise (http://www.aerolaser.es/).

\section{REFERENCES}

Aguilar, M.A., Aguilar, F.J. and Agüera, F., 2008. Assessing Geometric Reliability of Corrected Images from Very High Resolution Satellites. Photogrammetric Engineering \& Remote Sensing, 74(12), pp. 1551-1560.

Aguilar, M.A., Aguilar, F.J., Saldaña, M.M. and Fernández, I., 2012. Geopositioning accuracy assessment of GeoEye-1 Panchromatic and Multispectral imagery. Photogrammetric Engineering and Remote Sensing, 78(3), pp. 247-257.

Aguilar, M.A., Aguilar, F.J, Carvajal, M. and Agüera, F., 2007. Comparison of two commercial solutions for generating DSMs from QuickBird stereo imagery. In: 2007 Annual Conference of the Remote Sensing \& Photogrammetry Society (RSPSoc2007), Newcastle, UK, unpaginated CD-ROM.

Deilami, K. and Hashim, M., 2011. Very High Resolution Optical Satellites for DEM Generation: A Review. European Journal of Scientific Research, 49(4), pp. 542-554.

FGDC, 1998. "Geospatial Positioning Accuracy Standards Part 3: National Standard for Spatial Data Accuracy". URL: http://www.fgdc.gov/standards/projects/FGDC-standards-

projects/accuracy/part3/chapter3, U.S. Federal Geographic Data Committee, Reston, Virginia. (6 April 2011).

Fraser, C.S. and Ravanbakhsh, M., 2009. Georeferencing Accuracy of GeoEye-1 Imagery. Photogrammetric Engineering \& Remote Sensing, 75(6), pp. 634-638.

GeoEye, Inc., 2009. "GeoEye Product Guide". URL: http://www.geoeye.com/CorpSite/assets/docs/brochures/GeoEy e_Product_Guide.pdf (21 Dec. 2011).

Grodecki, J. and Dial, G., 2003. Block adjustment of highresolution satellite images described by rational polynomials. Photogrammetric Engineering \& Remote Sensing, 69(1), pp. $59-68$.

Kay, S. and Zielinski, R., 2007. Orthorectification and geometric quality assessment of Cartosat-1 for Common Agricultural Policy monitoring. In: Proc. ISPRS Workshop High-Resolution Earth Imaging for Geospatial Inf., Hannover, Germany, May 29 - Jun. 1, 2007.

JRC, Joint Research Centre, ISPRA, 2008. "Guidelines for Best Practice and Quality Checking of Ortho Imagery, v 3.0". URL: http://mars.jrc.it/mars/Bulletins-Publications/Guidelines-forBest-Practice-and-Quality-Checking-of-Ortho-Imagery-v-3.0 (6 April 2011).
Li, R., Zhou, F., Niu, X. and Di, K., 2007. Integration of Ikonos and QuickBird imagery for geopositioning accuracy analysis. Photogrammetric Engineering \& Remote Sensing, 73(9), pp. 1067-1074.

Meguro Y. and Fraser, C.S., 2010. Georeferencing accuracy of GeoEye-1 stereo imagery: Experiences in a Japanese test field. In: International Archives of the Photogrammetry, Remote Sensing and Spatial Information Science, Volume XXXVIII, Part 8, ISPRS Commission VIII, pp. 1069-1072.

Mitchell G. and MacNabb, K., 2010. High resolution stereo satellite elevation mapping accuracy assessment, In: Proceedings of the 2010 Annual ASPRS Conference, San Diego, California, 26-30 April, unpaginated CD-ROM.

Toutin, T., 1995. Multi-source data integration with an integrated and unified geometric modelling. EARSeL Journal Advances in Remote Sensing, 4(2), pp. 118-129.

Toutin, T., 2003. Error tracking in Ikonos geometric processing using a 3D parametric model. Photogrammetric Engineering \& Remote Sensing, 69(1), pp. 43-51.

Toutin, T., 2004. Comparison of stereo-extracted DTM from different high-resolution sensors: SPOT-5, EROS-A, IKONOSII, and QuickBird. IEEE Transactions on Geoscience and Remote Sensing, 42(10), pp. 2121-2129.

Wang, W. and Zhao L., 2011. Geolocation Accuracy Evaluation of GeoEye-1 Stereo Image Pair. In: International Symposium on Image and Data Fusion, ISIDF, Yunnan, China. Conference papers from IEEEXplore. 